\title{
Protein disulfide isomerase acts as an injury response signal that enhances fibrin generation via tissue factor activation
}

\author{
Christoph Reinhardt, ${ }^{1}$ Marie-Luise von Brühl,, ${ }^{2}$ Davit Manukyan, ${ }^{1}$ Lenka Grahl,, ${ }^{1}$ Michael Lorenz, ${ }^{2}$ \\ Berid Altmann, ${ }^{1}$ Silke Dlugai, ${ }^{1}$ Sonja Hess, ${ }^{3}$ Ildiko Konrad,, ${ }^{2}$ Lena Orschiedt, ${ }^{2}$ Nigel Mackman, ${ }^{4}$ \\ Lloyd Ruddock, ${ }^{5}$ Steffen Massberg, ${ }^{2}$ and Bernd Engelmann ${ }^{1}$
}

\begin{abstract}
${ }^{1}$ Vaskuläre Biologie und Hämostase, Institut für Klinische Chemie, Ludwig-Maximilians-Universität, Munich, Germany. ${ }^{2}$ Deutsches Herzzentrum, Munich, Germany. ${ }^{3}$ Proteome Exploration Laboratory, Beckman Institute, California Institute of Technology, Pasadena, California, USA. ${ }^{4}$ Department of Medicine, University of North Carolina School of Medicine, Chapel Hill, North Carolina, USA. ${ }^{5}$ Department of Biochemistry, Biocenter Oulu, Oulu, Finland.
\end{abstract}

\begin{abstract}
The activation of initiator protein tissue factor (TF) is likely to be a crucial step in the blood coagulation process, which leads to fibrin formation. The stimuli responsible for inducing TF activation are largely undefined. Here we show that the oxidoreductase protein disulfide isomerase (PDI) directly promotes TF-dependent fibrin production during thrombus formation in vivo. After endothelial denudation of mouse carotid arteries, PDI was released at the injury site from adherent platelets and disrupted vessel wall cells. Inhibition of PDI decreased TF-triggered fibrin formation in different in vivo murine models of thrombus formation, as determined by intravital fluorescence microscopy. PDI infusion increased - and, under conditions of decreased platelet adhesion, PDI inhibition reduced - fibrin generation at the injury site, indicating that PDI can directly initiate blood coagulation. In vitro, human platelet-secreted PDI contributed to the activation of cryptic TF on microvesicles (microparticles). Mass spectrometry analyses indicated that part of the extracellular cysteine 209 of TF was constitutively glutathionylated. Mixed disulfide formation contributed to maintaining TF in a state of low functionality. We propose that reduced PDI activates TF by isomerization of a mixed disulfide and a free thiol to an intramolecular disulfide. Our findings suggest that disulfide isomerases can act as injury response signals that trigger the activation of fibrin formation following vessel injury.
\end{abstract}

\section{Introduction}

Fibrin formation represents a major defense and repair mechanism, which ensures the integrity of the vascular system (1-3). Together with platelets, which adhere and aggregate at sites of vessel injury, fibrin formation is mandatory for stable sealing of the ruptured vessel wall and for prevention of life-threatening blood loss. Apart from its immediate actions necessary to arrest bleeding, fibrin also regulates the subsequent steps of wound healing. To accomplish its multiple functions only in cases of vascular wall damage, blood coagulation is functionally inactive in the intact vasculature but is rapidly triggered when the barrier function of the endothelium is perturbed. The initiation process of fibrin formation is known to crucially demand tissue factor (TF), a type I membrane protein predominantly resident in the cell membrane. TF, which is expressed by cells of the vascular wall, blood cells, and cell-derived microvesicles (or microparticles; ref. 4), promotes fibrin formation by increasing the proteolytic activity of the coagulation factor VIIa by several orders of magnitude. This requires the interaction of factor VIIa with the membrane-proximal region of the extracellular domain of TF.

Aberrant activation of blood coagulation is a major determinant of thrombotic occlusions of arteries and veins (4,5). Among other

Nonstandard abbreviations used: DTNB, 5,5'-dithio-bis(2-nitrobenzoic acid); GRX, glutaredoxin; GSH, reduced glutathione; LC-MS/MS, liquid chromatography-tandem mass spectrometry; MPB, 3-( $N$-maleimidylpropionyl)-biocytin; PDI, protein disulfide isomerase; $\mathrm{PDI}_{\mathrm{ox}}$, completely oxidized PDI; $\mathrm{PDI} \mathrm{red}_{\mathrm{red}}$, completely reduced PDI; sTF, soluble TF; TF, tissue factor; TRX, thioredoxin.

Conflict of interest: The authors have declared that no conflict of interest exists. Citation for this article: J. Clin. Invest. 118:1110-1122 (2008). doi:10.1172/JCI32376. complications, this may lead to arterial thrombosis resulting in myocardial infarction and stroke, which constitute the major causes of morbidity and mortality in most industrialized countries. While hemostasis and thrombosis are mechanistically different processes, TF is likely to contribute also to both arterial and venous thrombosis. Indeed, TF present in atherosclerotic arteries is supposed to be a major trigger for intravascular thrombus formation after rupture of atherosclerotic plaques $(4,5)$.

The coagulation start is thought to be maintained in a silent state by a condition that has been designated as TF encryption $(6,7)$, in which the protein is correctly inserted in the plasma membrane, but is functionally inactive. TF encryption has been documented for a vast number of cell types and for microparticles $(8,9)$. Several conditions modulate TF activity, including the membrane domain association of TF (10) and the phosphatidylserine transmigration to the outer leaflet of the plasma membrane (11). TF activity is also modulated by the redox state of its membrane proximal cysteine pair (Cys186/Cys209) (12), and oxidation of this cysteine pair increases the procoagulant function of TF. However, it is unclear which of these mechanisms is of relevance for TF activation during thrombus formation. Indeed, the nature of the stimuli that initiate fibrin generation after vessel injury in vivo and the way in which these stimuli convert TF from the encrypted to a functionally active state have remained largely undefined.

\section{Results}

Activation of coagulation by microparticle TF requires disulfide isomerases in vivo and in vitro. To study the mechanisms implicated in TF activation, we used extracellular membrane vesicles (microparticles), 

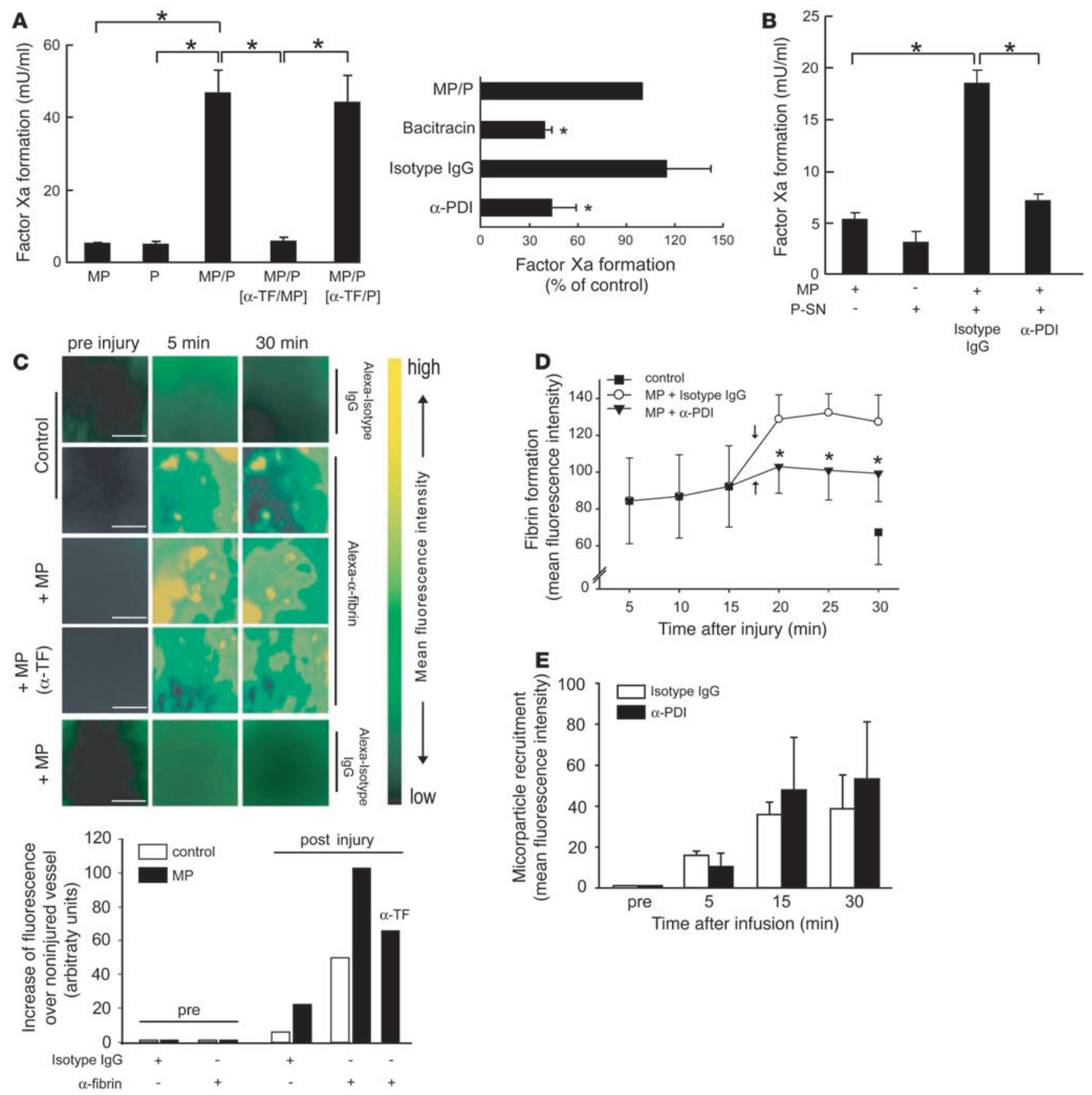

Figure 1

TF-dependent luminal fibrin generation in vivo is controlled by PDI. (A) Platelet-secreted disulfide isomerases activate microparticle TF. Left: Monocyte microparticles (MP; $\left.10^{6}\right)$ were incubated with collagen-activated platelets $\left(\mathrm{P} ; 5 \times 10^{7}\right)$. Microparticles $(\alpha-\mathrm{TF} / \mathrm{MP})$ or activated platelets $(\alpha-T F / P)$ were selectively preincubated with anti-TF antibody $(10 \mu \mathrm{g} / \mathrm{ml})$. Unbound antibody was removed by centrifugation. ${ }^{*} P<0.05$. Right: Microparticles were incubated with activated platelets for 10 minutes, then bacitracin, anti-PDI antibody, or control antibody were added to bypass inhibition of platelet stimulation. TF activity was determined by 2 -stage assay. ${ }^{*} P<0.05$ versus MP/P. Means \pm SD; $n=5$. (B) Plateletreleased PDI stimulates TF activity. Supernatants (P-SN) recovered from activated platelets $\left(10^{9}\right)$ were incubated with microparticles $\left(10^{6}\right)$ plus anti-PDI antibody or control antibody (15 minutes). The procoagulant activity (2-stage assay) was suppressed by anti-TF antibody. ${ }^{\star} P<0.05$ versus control antibody. $n=4$. (C) Microparticles accelerate fibrin production in mice. Microparticles (107; in $250 \mu \mathrm{l})$ were infused into WT mice after carotid ligation injury. MP ( $\alpha$-TF), microparticles precoated with $\alpha$-TF. Top: Fibrin (pseudocolored) was visualized by intravital videofluorescence microscopy using an Alexa Fluor 488-labeled anti-fibrin antibody. Measurements were performed before (pre injury) or 5 and 30 minutes after injury. Scale bar: $50 \mu \mathrm{m}$. Bottom: Quantitative analyses of the data. Means from 2 experiments, 30-minute values. (D) PDI contributes to microparticle-driven initiation of coagulation. Anti-PDI antibody (also recognizing murine PDI, $800 \mu \mathrm{g} / \mathrm{animal}$ ) or control IgG were infused into WT mice (15 minutes after injury), then microparticles $\left(10^{7}\right)$ were added (arrows). Fibrin formation at the injury site was monitored. ${ }^{*} P<0.05$ versus isotype IgG. $n=4-6$. (E) Microparticle recruitment is unchanged by PDI suppression. Anti-PDI antibody was infused 15 minutes after carotid injury. Then DCF-tagged microparticles were infused. Labeled microparticles at lesion site were measured by intravital microscopy. $n=3-4$. 

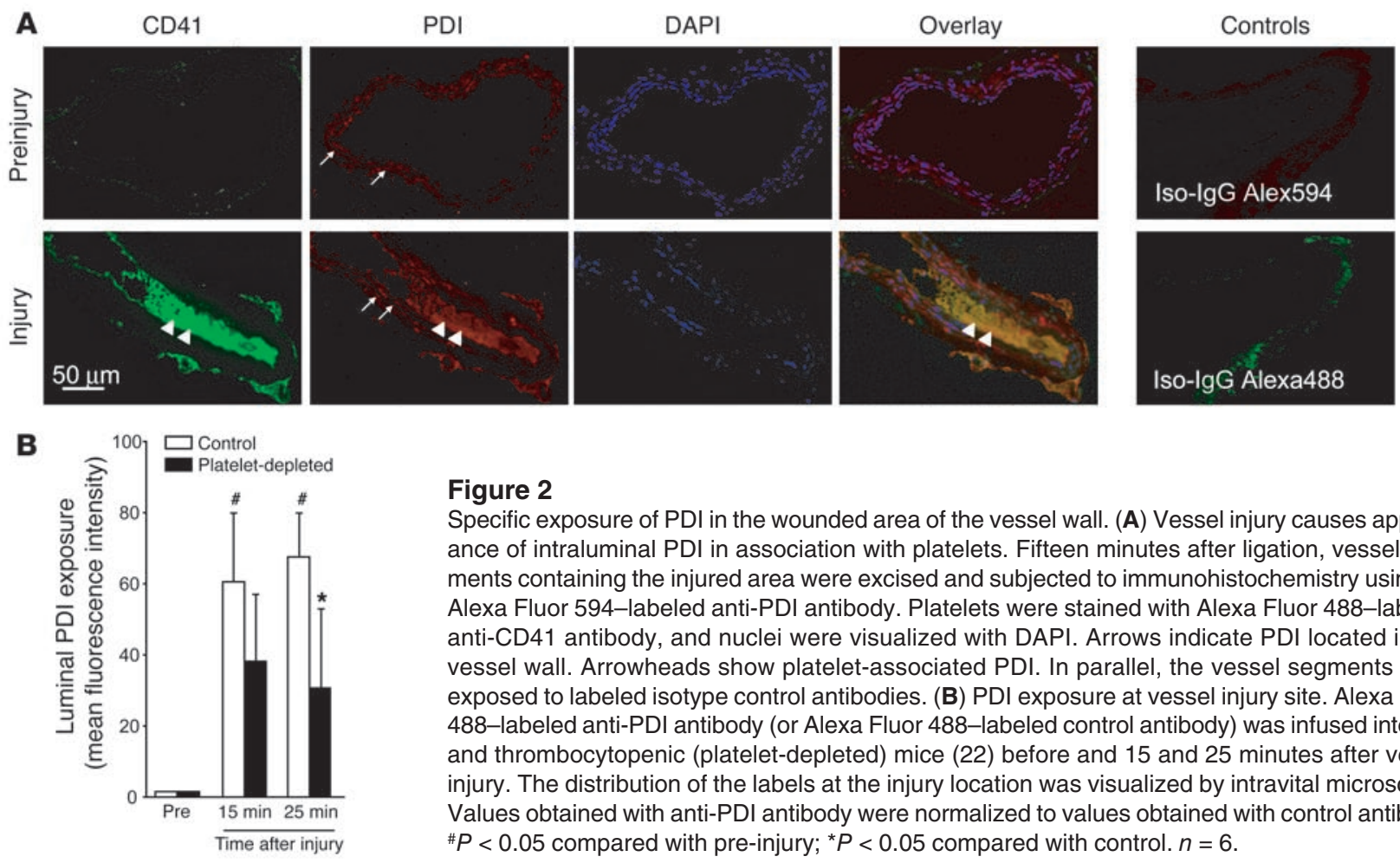

Figure 2

Specific exposure of PDI in the wounded area of the vessel wall. (A) Vessel injury causes appearance of intraluminal PDI in association with platelets. Fifteen minutes after ligation, vessel segments containing the injured area were excised and subjected to immunohistochemistry using an Alexa Fluor 594-labeled anti-PDI antibody. Platelets were stained with Alexa Fluor 488-labeled anti-CD41 antibody, and nuclei were visualized with DAPI. Arrows indicate PDI located in the vessel wall. Arrowheads show platelet-associated PDI. In parallel, the vessel segments were exposed to labeled isotype control antibodies. (B) PDI exposure at vessel injury site. Alexa Fluor 488-labeled anti-PDI antibody (or Alexa Fluor 488-labeled control antibody) was infused into WT and thrombocytopenic (platelet-depleted) mice (22) before and 15 and 25 minutes after vessel injury. The distribution of the labels at the injury location was visualized by intravital microscopy. Values obtained with anti-PDI antibody were normalized to values obtained with control antibody. ${ }^{\#} P<0.05$ compared with pre-injury; ${ }^{*} P<0.05$ compared with control. $n=6$.

which are major TF carriers in blood (9) and in atherosclerotic plaques (13). The TF activity of isolated monocyte-derived microparticles alone was low, indicating that microparticle TF was functionally mostly inactive (encrypted) (Figure 1A). Microparticles coadhere with platelets at vascular lesions $(14,15)$, and this interaction could be involved in the activation of microparticle TF (16). Indeed, the procoagulant activity of microparticle TF was amplified substantially by activated platelets (Figure 1A). Activated platelets are known to release disulfide isomerases (17), which regulate thiol modifications. To test the potential participation of these enzymes in the activation of microparticle TF, we used bacitracin, a broad inhibitor of the oxidoreductase protein disulfide isomerase (PDI) and other disulfide isomerases (17). Bacitracin partially prevented activation of microparticle TF by activated platelets (Figure 1A). In addition, selective suppression of PDI by an anti-PDI antibody attenuated TF activation induced by activated platelets (Figure 1A). The control antibody was without effect. Inhibition of PDI also suppressed stimulation of microparticle TF when intact platelets were substituted by platelet releasates (Figure 1B). Platelet-released PDI thus contributes to activation of microparticle TF independent of the presence of the activated platelets themselves.

To address the role of PDI in the microparticle-induced initiation of coagulation in vivo, vascular injury was induced by ligation of the carotid artery (18). Using a labeled anti-fibrin antibody, fibrin formation at the injury site was visualized in real time by high-speed intravital fluorescence microscopy (Figure 1C). Experiments with a labeled control antibody verified the specificity of the signal. Infusion of microparticles into the jugular vein of WT mice increased fibrin generation on the thrombus surface (Figure 1C). Microparticle TF was confirmed as trigger of coagulation, since infusion of microparticles coated with anti-TF antibody largely failed to increase the fibrin formation (Figure 1C), extending previous work (19). Next, to inhibit PDI in vivo, the anti-PDI antibody (or control antibody) was infused into the murine blood circulation before infusion of microparticles. In the presence of control antibody, microparticles increased fibrin formation (Figure 1D), in agreement with the results of Figure 1C. In contrast, inhibition of PDI suppressed the coagulation activation in the presence of infused microparticles. This was not due to an effect of PDI inhibition on microparticle attachment per se (Figure 1E).

Then we visualized the localization of PDI in the surroundings of the vessel injury site. Using immunohistochemistry, we detected intraluminal accumulation of PDI at the site of the vessel lesion, in particular on the surface of thrombus-associated platelets (Figure 2A). Furthermore, PDI was located in the vessel wall (Figure 2A), in particular in association with vascular smooth muscle cells, as indicated by costaining with smooth muscle actin (not shown). In the unperturbed vasculature, the localization of PDI was restricted to the vessel wall (Figure 2A). The labeled control antibodies yielded negligible staining, mostly of the vessel adventitia. Luminal exposure of PDI at the injury site was confirmed by intravital microscopy (Figure 2B). Again, no intraluminal localization of PDI was detected in the intact vessel. The PDI exposure in injured vessels could originate from activated platelets and, additionally, from the ER of disrupted cells of the vessel wall. The latter origin is a likely possibility, since PDI is present in the millimolar range within the ER $(20,21)$. Moreover, experiments with mechanically wounded CHO cells (cell scraping) in vitro indicate that cell injury induces the release of PDI together with other ER lumenal proteins (e.g., GRP94 and GRP78; data not shown). To distinguish between platelet- and vessel wall-derived PDI, we induced massive thrombocytopenia by targeting platelet GPIb $\alpha$ with an antibody mixture that suppresses platelet counts by more than $95 \%$ (22). In 

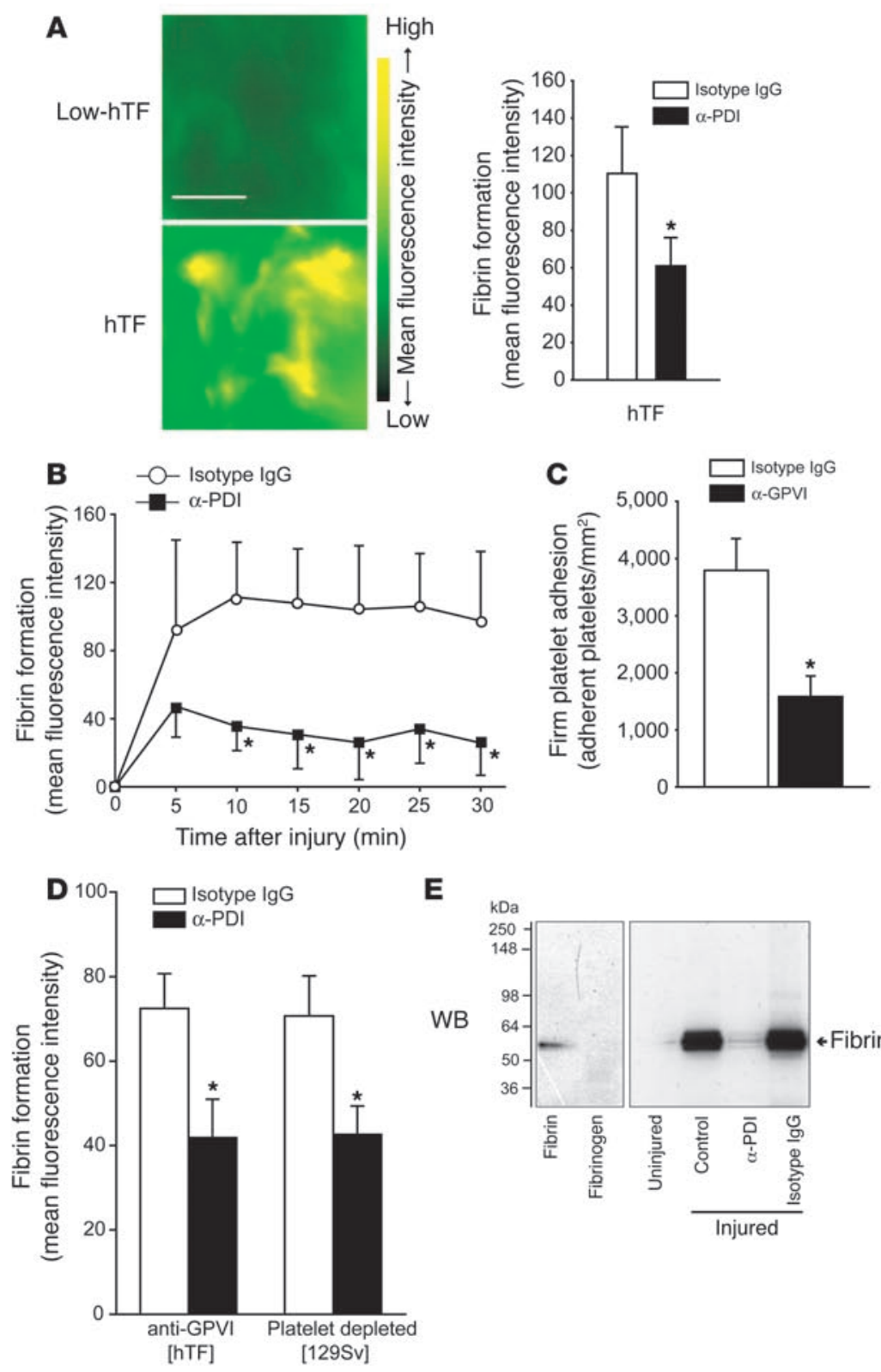

E

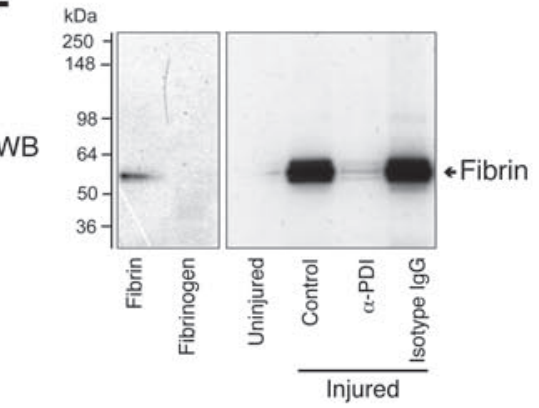

Figure 3

PDI contributes to trigger TF-dependent fibrin formation under conditions of suppressed platelet adhesion. (A) TF-dependent initiation of coagulation in living animals. Left: Fibrin formation was assessed 15 minutes after carotid artery ligation by intravital microscopy in hTF mice and low-hTF mice. Scale bar: $50 \mu \mathrm{m}$. Right: Quantitation of fibrin formation at site of vessel injury. Effect of anti-PDI antibody (or control antibody; infused before implementing the vessel injury) on fibrin formation in hTF animals. ${ }^{*} P<0.05$ versus isotype control. $n=4$. (B) PDI contributes to fibrin formation in mice with normal platelet counts. Fibrin generation was assessed before or after vascular injury (ligation) in WT (SV129S1) mice pretreated with the indicated antibodies prior to induction of vessel injury. ${ }^{*} P<0.05$ versus isotype control. $n=4$. (C) GPVI inhibition decreases platelet adhesion. Firm platelet adhesion of rhodamine-labeled platelets to the injured artery (ligation) was visualized 15 minutes after injury in the presence of control antibody or neutralizing anti-GPVI antibody (hTF mice). ${ }^{*} P<0.05 . n=8$. (D) PDI regulates fibrin formation under conditions of decreased platelet adhesion. Platelet adhesion was inhibited by infusion of anti-GPVI antibody (hTF mice). Thrombocytopenia was induced by injection of anti-GPIb $\alpha$ antibody (platelet-depleted WT mice). Both groups were then treated intravenously with anti-PDI antibody or control antibody, vessels were injured, and fibrin formation was determined after 15 minutes. ${ }^{*} P<0.05$ versus isotype control. $n=7-10$. (E) PDI increases fibrin formation after wire-induced vessel injury. Fibrin formation after wire-induced injury of the murine carotid artery was assessed by western blotting in the presence of $\alpha$-PDI or control IgG. The anti-fibrin antibody specifically detects fibrin but not fibrinogen. Thrombi were excised 15 minutes after vascular injury. thrombocytopenic mice, we observed PDI exposure at the injury site by intravital microscopy 15 and 25 minutes after vessel injury (Figure 2B). After 30 minutes, release of PDI was decreased in these mice compared with control mice, suggesting that the luminal exposure of PDI in WT mice at this time point is mostly derived from platelets. Together this indicates that the PDI exposed at the site of vessel injury not only originates from activated platelets but also from the disrupted vessel wall cells. In summary, PDI selectively exposed at sites of vascular lesions is required for TF-triggered fibrin generation.

PDI triggers TF-dependent blood coagulation in mice. We then determined whether PDI is involved in fibrin formation in the absence of exogenously applied microparticles. We monitored fibrin generation in mice expressing human TF (hTF mice; ref. 23) at levels comparable to its murine counterpart in WT mice. In hTF animals there was extensive fibrin production at the injury site (ligation injury; Figure 3A). In contrast, fibrin formation was minimal in mice with low hTF (24). Hence hTF mice enabled analysis of hTFdriven fibrin generation in vivo. To suppress the PDI activity in the vessel wound, the anti-PDI antibody was infused into the hTF animals prior to induction of vessel injury. Inhibition of PDI substantially reduced fibrin formation at the injury site (Figure 3A), which was accompanied by a decrease in the total area occupied by fibrin. Moreover, experiments performed in WT mice showed that blockade of PDI substantially attenuates fibrin formation following endothelial disruption (Figure 3B). Nonetheless, preliminary experiments indicate that the tail bleeding times in WT mice treated with anti-PDI antibody are not significantly prolonged compared with treatment with control IgG $(1428 \pm 61 \mathrm{~s}$ [anti-PDI] vs. $996 \pm 208 \mathrm{~s}$ [control IgG]; mean \pm SEM, $n=5$ animals per treatment). To test whether the influence of the antibody was due to a strong inhibitory effect on platelet adhesion at the injury site, 

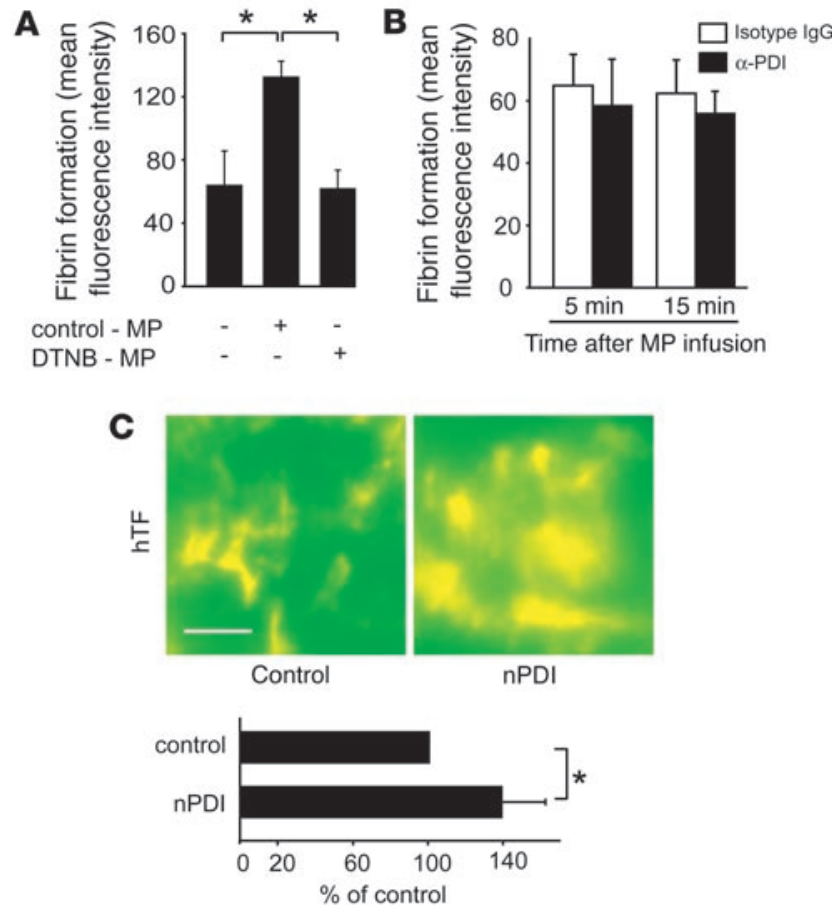

we suppressed platelet adhesion by blocking the platelet collagen receptor GPVI (Figure 3C). This did not influence blood platelet counts $\left(644 \pm 80 \times 10^{3} / \mu\right.$ l before injection and $584 \pm 46 \times 10^{3} / \mu \mathrm{l}$ after injection of anti-GPVI antibody; mean \pm SEM, $n=4-6)$. In hTF mice with decreased platelet adhesion, fibrin formation at the injury site was lowered (Figure 3D versus Figure 3A). This confirms that platelet adhesion/activation contributes to fibrin generation following vascular injury. However, also under conditions of decreased platelet adherence, fibrin formation was decreased following infusion of anti-PDI antibody (Figure 3D). Moreover, in thrombocytopenic WT mice, fibrin formation at the vessel injury site was diminished by PDI inhibition (Figure 3D). Together our findings indicate that PDI inhibition lowers fibrin formation both in the presence of platelet adhesion and under conditions of suppressed platelet adherence. This suggests that PDI released from disrupted vessel wall cells (Figure 2B) also contributes to initiation of fibrin formation. To determine whether PDI-driven initiation of coagulation is also relevant in another model of thrombus formation, the endothelium of the carotid artery was denudated by a wire device (18). Subsequently, fibrin generation was determined by immunoblotting of isolated thrombi in WT mice. Also fibrin formation in response to wire injury was substantially decreased by suppression of PDI (Figure 3E).

To evaluate whether TF disulfide exchange contributes to PDImediated fibrin formation in the intact animal, microparticles were pretreated with the free thiol-selective reagent $5,5^{\prime}$-dithiobis(2-nitrobenzoic acid) (DTNB) before infusion of microparticles into the murine blood. This prevented the acceleration of fibrin production by microparticle-derived TF in the ligation model (Figure 4A). Next, the anti-PDI antibody was infused into the animals following injection of DTNB-pretreated microparticles to inhibit the interaction of PDI with microparticle TF. As a result, fibrin formation at the lesion site was no longer diminished by the anti-PDI antibody (Figure 4B), in contrast to its inhibitory effect after infu-

\section{Figure 4}

TF disulfide exchange supports fibrin production in vivo. (A) Prevention of TF disulfide switching inhibits fibrin generation. Monocyte microparticles were preincubated for 30 minutes ex vivo with DTNB (1 mM; DTNB-MP) or vehicle (control-MP). Then they were infused into the murine blood. Fibrin generation at the lesion site (ligation injury) was determined 15 minutes after infusion of microparticles. ${ }^{*} P<0.05 . n=4$. (B) PDI inhibition requires functioning disulfide exchange in vivo. The anti-PDI antibody (or control antibody) was infused following incorporation of DTNB-pretreated microparticles into the murine blood circulation. Fibrin generation at the lesion site (ligation injury) was determined 15 minutes after infusion of microparticles. $n=4$. (C) TF-driven fibrin formation is enhanced by PDI. PDI ( $200 \mu \mathrm{g} / \mathrm{animal})$ was included in the blood circulation of hTF mice, and fibrin generation was registered 10 minutes after vessel injury at the lesion site (ligation injury). Scale bar: $50 \mu \mathrm{m} .{ }^{*} P<0.05 . n=6$.

sion of untreated microparticles under similar conditions (data not shown). This indicates that the anti-PDI antibody inhibits the PDI-mediated thiol-disulfide exchange and decreases the activation of TF in vivo. In a complementary approach, native PDI was added to the circulation of the hTF animals prior to induction of vessel injury. This augmented the focal fibrin generation (Figure $4 \mathrm{C}$ ), indicating that exogenous enzyme can enhance the effect of endogenous PDI. Overall, the results from different models of thrombogenesis suggest that fibrin generation at the lesion site is supported by PDI-mediated disulfide exchange of TF.

PDI induces disulfide bond formation in TF. To evaluate how activated platelets and PDI might activate TF, we tested their effects on the sulfhydryl status of TF. The extracellular TF domain (soluble TF [sTF]; lacking the transmembrane and cytoplasmic domains) was labeled with 3-( $N$-maleimidylpropionyl)-biocytin (MPB), which selectively reacts with free thiols (25). In folded STF (see Methods), only the C186/C209 pair is accessible to solvents $(26,27)$. After exposure of sTF to supernatants from activated platelets, the number of free thiols of sTF was decreased (Figure 5A), consistent with disulfide formation. Cell lysate, another TF activator, also diminished TF free thiols (data not shown). Furthermore, PDI decreased the number of free thiols in STF (Figure 5A). In contrast, the reductase thioredoxin (TRX) slightly increased the TF free thiols. Western blots performed in parallel excluded that the different experimental conditions and the MPB labeling affected recognition of STF by the anti-TF antibody (Figure 5A). The densities of the bands obtained by MPB labeling of sTF plus subsequent pulldown with streptavidin agarose (indicated as MPB-IB in Figure 5A) cannot be directly compared with those obtained by direct western blotting of MPB-labeled STF and hence cannot be used for quantitative determination of the amount of TF containing free thiols because of the different procedures employed (see also legend to Figure 5A). We next assessed the extracellular free thiols in full-length TF expressed in human monocytes, using MPB concentrations that were not associated with cell internalization of the probe. Free thiol groups were detected in cell surface TF. These were diminished by PDI (Figure 5B).

Constitutive glutathionylation of TF contributes to maintaining a low TF activity. To further specify thiol modifications of TF, we assessed whether cell TF was glutathionylated, which represents a major thiol modification of several proteins containing redox-active cysteines (28). Glutathionylated proteins in cell lysates of monocytes and glutathionylated cell surface proteins in intact cells were 

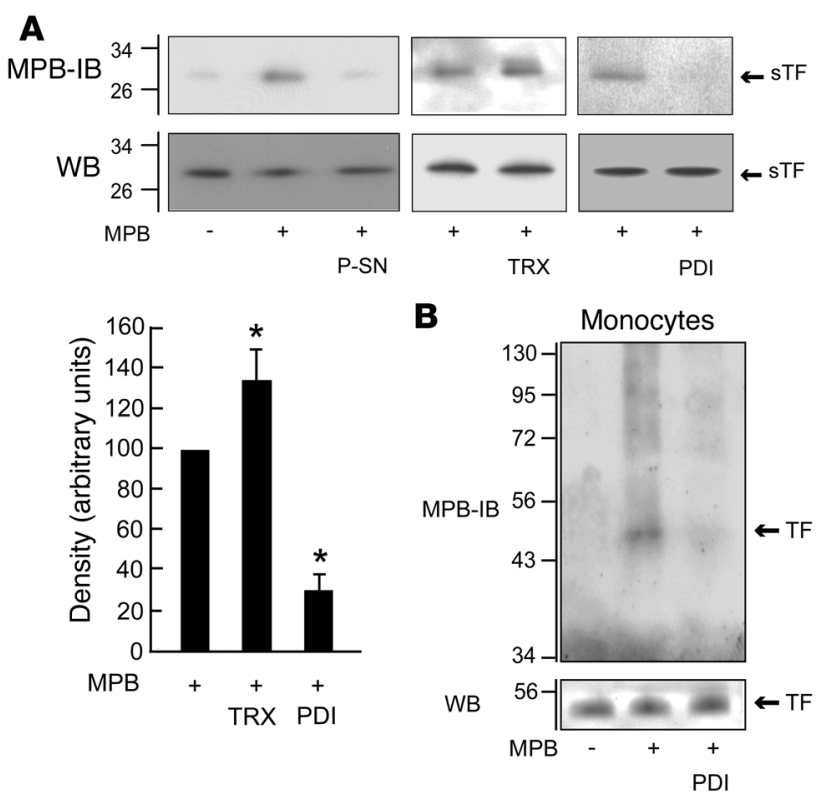

immunoprecipitated with an anti-glutathione antibody. To assess whether TF was among the glutathionylated proteins, this was followed by TF detection on western blots using an anti-TF antibody. The results show that full-length TF in cell lysates (Figure $6 \mathrm{~A}$ ) and on the cell surface (data not shown) contains mixed disulfides with glutathione. In parallel experiments we found that exposure of sTF labeled with biotin-glutathione (Figure 7A) to cell lysates from $\mathrm{CHO}$ cells decreased TF glutathionylation (data not shown), suggesting that the amount of glutathione conjugated to TF might be underestimated by the IP experiments (Figure 6A), which mandatorily require cell lysis.

To further detail the presence of mixed disulfides in the extracellular TF domain, sTF was subjected to liquid chromatography-tandem mass spectrometry (LC-MS/MS). While sTF showed some heterogeneity, the major peak of the protein had a mass consistent with a single glutathionylation event. This peak represented $50 \%-60 \%$ of all detectable peaks. Single digestion of sTF with trypsin, GluC, or AspN yielded only poor sequence coverage. However, after double digestion with trypsin, tryptic and semitryptic peptides were obtained yielding a sequence coverage of $68 \%$ (Supplemental Table 1; supplemental material available online with this article; doi:10.1172/JCI32376DS1). For the C49- and C57-containing peptides, the experimentally obtained masses were about $2 \mathrm{Da}$ lower than the calculated masses (based on the assumption that both cysteines were reduced). This is consistent with a loss of $2 \mathrm{H}$ atoms due to disulfide bond formation between the $\mathrm{N}$-terminal cysteines. All peptides containing C209 were found with a 305.1-Da mass shift to their predicted masses, consistent with glutathionylation of C209. This does not exclude the presence of other thiol modifications at C209 that might have escaped detection, such as other types of mixed disulfides and/or intramolecular disulfides between C186 and C209. To definitely clarify the structure of the C209-containing peptides, they were subjected to MS/MS fragmentation. As shown for the peptide 202-214 (Figure 6B), this induces cleavage of the peptide bonds between the individual amino acids of this peptide and thus generates peptide fragments of all possible chain lengths. The differ-

\section{Figure 5}

PDI decreases TF free thiols. (A) PDI lowers TF free thiols. Top panels: STF $(1.7 \mu \mathrm{g} / \mathrm{ml})$ was exposed for 15 minutes to platelet releasates from collagen-activated platelets $\left(5 \times 10^{8}\right)$, TRX $(10 \mu \mathrm{M})$, or PDI $(1.8 \mu \mathrm{M})$. In all cases, STF was then incubated with biotin-MPB. One aliquot was then pulled down with streptavidin agarose, and subsequently the biotinylated proteins were detected by immunoblotting with anti-TF antibody (MPB-IB). Another aliquot of the STF previously incubated with MPB was directly subjected to western blotting. Bottom panels: Densitometric analyses of the effects of TRX and PDI on STF free thiols. ${ }^{*} P<0.05$. $n=4$. (B) PDI causes disulfide formation of cell TF. TF expressing monocytes $\left(10^{6}\right)$ were incubated for 15 minutes with PDI or vehicle. The cells were subsequently washed and then incubated with biotin-MPB. The biotinylated proteins were selected with streptavidin-agarose. Biotinylated TF species were detected by immunoblotting with anti-TF antibody. Western blots were performed under identical conditions.

ences in masses between the C209-containing peptide fragments $b_{7}$ and $b_{8}$ and between the C209-containing fragments $y_{6}$ and $y_{5}$ yielded values of 305.07. This corresponds to the mass difference between reduced cysteine (103.01) and glutathionylated cysteine (408.08) and thus establishes the presence of glutathione at C209. Similar results were obtained when the peptides 202-210 and 202212 were fragmented. Thus C209 in sTF is constitutively conjugated with glutathione.

To analyze the effect of glutathionylation on TF procoagulant activity, monocyte TF was first activated by cell lysis, and then DTT $(0.1 \mathrm{mM})$ was added to reduce part of the redox-active cysteines of TF. This DTT concentration was chosen because higher DTT concentrations by themselves completely inhibited TF activity of the cell lysates (Figure 6C; see below). Subsequently, reduced glutathione (GSH) was added in the presence of diamide to convert the reduced cysteines into mixed disulfides with glutathione (see legend to Figure 6C). As a consequence of experimental TF glutathionylation, the TF activity was decreased (Figure 6C). Control experiments were performed with GSH alone or DTT plus GSH. This did not alter TF activity. In the presence of higher DTT concentrations ( $1 \mathrm{mM}$ ) alone, which were verified not to alter TF folding (see Methods), TF activation by cell lysis was almost completely blocked (Figure 6C). This suggests that TF activation by cell lysis occurs via a redox-dependent mechanism. The results moreover indicate that glutathionylation contributes to TF encryption.

PDI converts TF cysteines from glutathionylated to disulfide state. PDI has 2 redox-active CXXC sites, which can exist in dithiol or disulfide states and which determine the type of redox reactions PDI can catalyze. The predominant redox state of PDI in vivo is the reduced dithiol form (29). Similarly, we found that the majority of the redox-active cysteines of the recombinant PDI used here were in the reduced state (see Methods). Since PDI is capable of catalyzing deglutathionylation reactions (30), we tested the effect of isolated PDI on TF glutathionylation. sTF was labeled with biotinglutathione (Figure 7A). In control experiments we verified that the linkage of labeled glutathione with TF could be disrupted by DTT and GRX (Figure 7A), in line with their ability to deglutathionylate proteins (28). PDI partially deglutathionylated TF (Figure 7B). The ability to cleave the glutathione linkage of sTF was slightly enhanced when the redox-active cysteines of PDI were completely reduced with DTT $\left(\mathrm{PDI}_{\mathrm{red}}\right)$. By contrast, after complete oxidation of these cysteines $\left(\mathrm{PDI}_{\mathrm{ox}}\right.$ ) with oxidized glutathione (GSSG), $\mathrm{PDI}_{\mathrm{ox}}$ did not alter TF glutathionylation, indicating that PDI needs to be in the reduced state to cleave the glutathione linkage. 
A

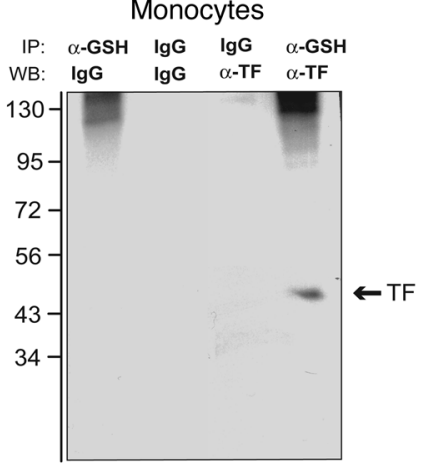

C

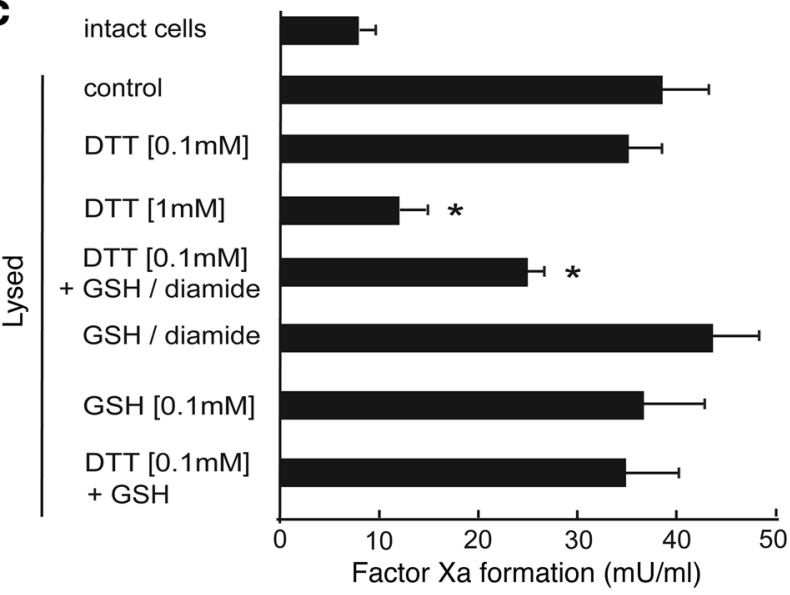

B

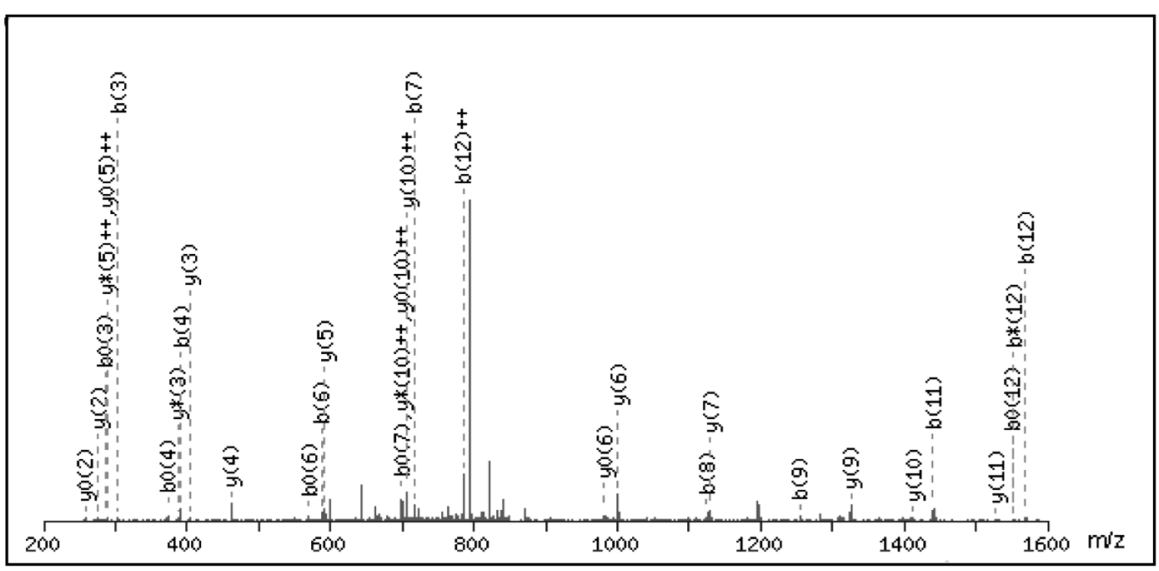

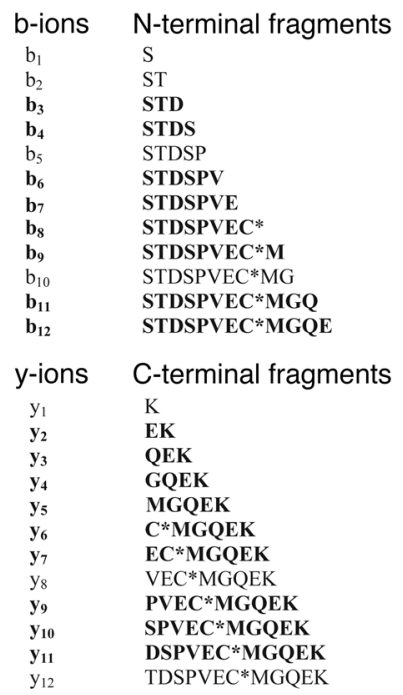

\section{Figure 6}

Constitutive glutathionylation of TF. (A) Basal glutathionylation of cell TF. Solubilized monocytes were immunoprecipitated with anti-GSH antibody or with isotype control antibody, as described in Methods. This was followed by western blotting with control antibody or anti-TF antibody. (B) Structure of C209 containing peptide STDSPVEC*MGQEK as identified by MS/MS fragmentation. The spectrum was generated by collisioninduced dissociation (CID) of the doubly charged 858.33- $\mathrm{m} / \mathrm{z}$ ion. CID of doubly charged precursor ions predominately forms singly charged product ions that are indicative of the sequence of a peptide. The product ions thus generated are $\mathrm{b}$-ions from the $\mathrm{N}$-terminal site and $\mathrm{y}$-ions from the C-terminal site. Peptide fragments observed in the mass spectrometer are shown in bold. The mass difference between $b_{7}$ and $b_{6}$ ions shows that the cysteine contains a modification equal to a glutathionylation from the N-terminal site. This is confirmed by the same mass difference between the $y_{5}$ and $y_{6}$ ion from the C-terminal site. (C) Glutathionylation attenuates TF activation. Cell lysis increases procoagulant activity, relative to intact cells, which is completely inhibited by anti-TF antibody. Increasing concentrations of DTT suppress TF activity. Glutathionylation in lysed cells was induced by preincubation with DTT $(0.1 \mathrm{mM})$, followed by incubation with GSH $(0.1 \mathrm{mM}$ plus $0.1 \mathrm{mM}$ diamide; 15 minutes). This increased the density of the band representing glutathionylated TF in immunoblots of lysed monocytes by 5.5-fold. Procoagulant activity of the lysates was determined by coagulation factor concentrate. ${ }^{*} P<0.05$ versus control. $n=3-9$.

To evaluate the consequences of the deglutathionylation for the free thiol status of TF, we determined MPB labeling of sTF in the presence of different PDI species. PDI and $\mathrm{PDI}_{\mathrm{red}}$, but not $\mathrm{PDI}_{\mathrm{ox}}$, decreased the free thiol content of STF (Figure 7C), consistent with the formation of intramolecular disulfides of TF. In contrast, GRX, which also deglutathionylates TF (Figure 7A), increased the TF free thiols (data not shown). This shows that PDI $_{\text {red }}$ does not deglutathionylate TF to the free thiol as does GRX. Instead, PDI in its reduced form is likely to catalyze an isomerization reaction from a free thiol and a glutathionylated thiol to the C186/C209 disulfide (see Discussion).
Next we assessed the influence of PDI on the procoagulant activity of TF. Under basal conditions, monocyte TF was largely inactive. However, PDI markedly increased monocyte TF activity (Figure 8A). This effect was completely suppressed by the anti-TF antibody (Figure 8A). In contrast to PDI, GRX (from E. coli, in the presence of GSH to maintain GRX in the fully reduced state) and TRX, which both reduce the redox-active cysteines of TF, did not enhance TF activity (Figure 8A). PDI (1.8 $\mu \mathrm{M}, 15$-minute incubation) also increased the procoagulant activity of sTF $(25 \mathrm{nM})$, while TRX $(10 \mu \mathrm{M})$ did not alter the activity of STF (control: $100 \%$, PDI: $208 \% \pm 9 \%, P<0.05$; TRX: $105 \% \pm 38 \% ; n=4$, 2 -stage assay). To test 
A

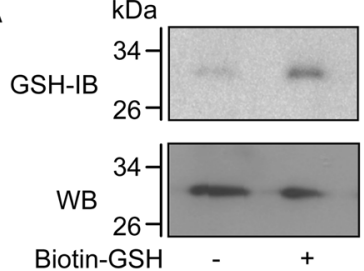

B
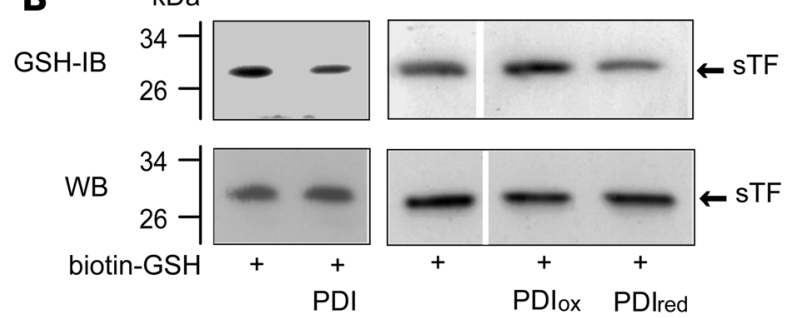

C

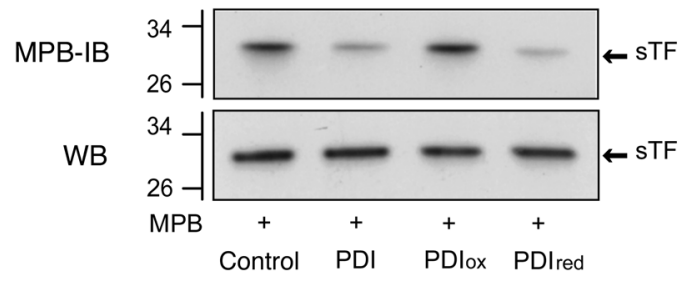

Figure 7

Deglutathionylation and TF disulfide bond formation by reduced PDI. (A) Incorporation of glutathione into STF is reverted by GRX and DTT. STF $(1.7 \mu \mathrm{g} / \mathrm{ml})$ incorporates biotin-labeled glutathione $(0.1 \mathrm{mM}$, in the presence of $0.1 \mathrm{mM}$ diamide; 15 minutes). Where indicated, DTT $(1 \mathrm{mM})$ and $\mathrm{GRX}(4 \mu \mathrm{M}$; in the presence of $1 \mathrm{mM} \mathrm{GSH})$ were added together with biotin-labeled glutathione. The biotinylated proteins were captured by streptavidin-agarose and immunoblotted with anti-TF antibody (GSH-IB). In parallel, sTF was analyzed by western blotting. (B) Deglutathionylation of TF by PDI. Left: STF was labeled with biotin-labeled glutathione in the absence (control) or presence of native PDI, fully oxidized PDI (PDI $1.8 \mu \mathrm{M}, 15$ minutes). Biotinylated proteins selected by streptavidin-agarose were immunoblotted with anti-TF antibody, and in parallel, sTF was analyzed by western blotting. Right: Quantification of labeling of STF with biotin-glutathione by densitometry. ${ }^{*} P<0.05$ (versus control). $n=4$. (C) Reduced forms of PDI promote disulfide bond formation of TF. Left: sTF was exposed to vehicle (control) and native PDI, PDI ox $_{\text {, or PDI }}$ (all at $1.8 \mu \mathrm{M}, 15$ minutes, room temperature). Then, the samples were incubated with biotin-MPB. The biotinylated proteins were selected with streptavidin-agarose, followed by detection of biotinylated STF species by immunoblotting with anti-TF antibody. In parallel, western blots were performed. Right: Quantification of free thiols of sTF by densitometry. ${ }^{*} P<0.05$ versus control.

whether the redox-active cysteine CXXC sites of PDI are required for TF activation, we used a PDI mutant in which all cysteines are replaced by serine (PDI $\Delta$ Cys 1,2$)$. This mutant lacks the oxidoreductase activity of PDI but retains its chaperone function (44). In contrast to native PDI, PDI $\Delta$ Cys 1,2 did not enhance TF activity (Figure 8B). Furthermore, PDI $\Delta$ Cys 1,2 did not share the ability of native PDI to deglutathionylate TF (Figure 8B). To reveal whether the thiol status of the redox-active cysteines of PDI is of relevance for its ability to activate TF, we tested the effects of $\mathrm{PDI}_{\text {red }}$ and $\mathrm{PDI}_{\mathrm{ox}}$ on the activity of cell TF. PDI $\mathrm{I}_{\text {red }}$ augmented TF activity to a comparable degree as underivatized PDI, while $\mathrm{PDI}_{\mathrm{ox}}$ was considerably less effective (Figure 8B). Hence activation of TF requires the presence of the redox-active cysteines of PDI and is most efficient when these cysteines are in the reduced form. Together, our results suggest that PDI can activate TF by an isomerization reaction, which proceeds via mixed disulfide cleavage and subsequent disulfide bond formation.

\section{Discussion}

Our study suggests that exposure of the oxidoreductase PDI at vessel lesions contributes to stimulate the crucial first step of blood coagulation, activation of the trigger protein TF. This indicates that disulfide isomerases may be major initiators of fibrin formation, which can convert TF from the functionally silent (or encrypted) state to the active form (Figure 9). In addition to other mechanisms, such as exposure of phosphatidylserine (10), PDI can indeed directly promote TFdependent fibrin formation. We identify constitutive linkages of TF Cys 209 with glutathione that maintain TF in a status of low procoagulant functionality. The redox-active cysteines of intracellular and cell-released PDI are predominantly present as free thiols $(29,31)$. Moreover, we found that TF is more efficiently activated by reduced PDI than by oxidized PDI. Since PDI decreases the free thiols of TF, it activates TF by a specific type of disulfide exchange that allows the reduced enzyme to induce the formation of intramolecular disulfides in TF. This suggests that PDI can initiate blood coagulation through isomerization of the Cys186/ Cys209 pair of TF (Figure 9).

This could potentially proceed via the generation of an unstable mixed disulfide between the $\mathrm{N}$-terminal active site cysteine of PDI and Cys209 of TF, followed by a nucleophilic attack by Cys 186 on this intermolecular disulfide. The alternative product of the first step, the formation of a PDI-GSH mixed disulfide, is not favored (30). Apart from the glutathionylated forms, the TF cysteines might assume additional redox states. Overall, mixed disulfides are apparently critical for the TF activation via reduced PDI, as they provide the redox equivalents required for generation of the Cys186/Cys209 disulfide. Since an increasing number of proteins are characterized as substrates for glutathionylation (28) and PDI can regulate the functionality of several proteins via fast redox switching (such as, for example, platelet surface receptors and MHC class I molecules; refs. 17, 32), the type of disulfide switch promoted by PDI as identified here is likely to be of general relevance. Moreover, the findings suggest for the first time a major relevance for the posttranslational mechanism of disulfide switching under in vivo conditions.

The nature of the TF activation mechanism described implies that the redox status of the thiol isomerase itself remains unaltered after its interaction with TF. Consequently relatively few 


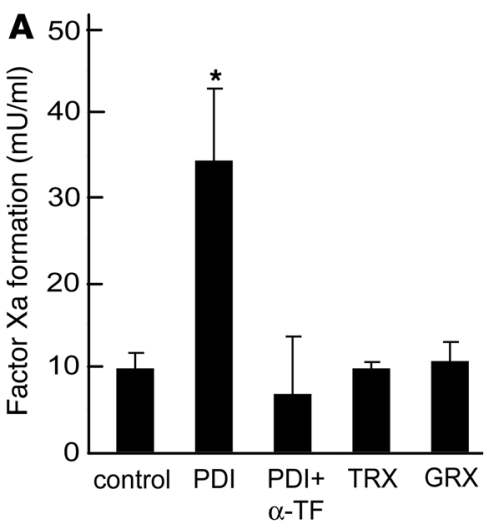

PDI molecules can in principle activate a substantial number of TF molecules. This will enable amplification of the vessel injury signal, which is required to promote rapid and optimal resealing of the vessel wound. Among the different redox modifications of TF so far identified, the Cys186/Cys209 intramolecular disulfide state is associated with TF activation. Our findings indicating that PDI activates TF via its oxidoreductase activity (see above) do not exclude the possibility that the enzyme might stimulate TF activity through additional mechanisms (33) or that under certain conditions it might decrease TF activity via $S$-nitrosylation-dependent mechanisms (12). In intact cells, PDI is mostly expressed in the ER $(20,21,34)$. After vascular injury, PDI is abundantly exposed on the luminal surface of the wounded vessel wall. The exposure is injury specific, since no PDI is detected on the surface of the intact vessel wall. The PDI released originates from adherent platelets and, additionally, from disrupted vessel wall cells, since PDI release at the vessel injury site is also seen in thrombocytopenic mice. PDI released from both sources is proposed to act in concert to initiate coagulation (Figure 9). Future work will be required to exactly dissect the role of the different sources of PDI released at the injury site that trigger TF-dependent fibrin formation and the participation of PDI-mediated amplification of platelet activation. Nonetheless, since PDI is exposed at the vessel lesion in thrombocytopenic mice, PDI inhibition decreases fibrin generation in mice with suppressed platelet adhesion, and since PDI stimulates the TF activity in isolated cells and microparticles, our findings suggest that under the experimental conditions applied the oxidoreductase directly activates TF in vivo and in vitro.

Disulfide exchange reactions have been described for a number of proteins in cellular systems (35-38). Our study indicates a major relevance for disulfide switching in the regulation of vessel resealing. Several central aspects of the molecular machinery activating TF in vitro can indeed be recaptured in the intact organism, including the mediator function of PDI and the participation of TF thiol-disulfide exchange. We document activation of TF-dependent blood coagulation via PDI by intravital monitoring of the dynamics of fibrin formation at the site of vessel injury. PDI is found to promote fibrin generation both during the early phase of thrombus development (10-15 minutes post injury) and at later stages, when fibrin generation is also driven by microparticle TF, a circulating carrier for TF (9) that is selectively recruited to the growing thrombus $(14,15)$. In particular, the activity of microparticle TF is shown to be uncovered by platelet-secreted PDI.

Spontaneous rupture of endothelial cells covering atherosclerotic plaques initiates arterial thrombosis in humans in which blood coagulation is aberrantly activated $(39,40)$. Plaque rupture and consecutive thrombosis represent key mechanisms in the pathogenesis of cardiovascular diseases such as myocardial infarction and stroke. In the thrombosis models utilized in the present study, local fibrin generation is equally induced by disruption of the endothelial surface, whereby substantial platelet accumulation is triggered. Consequently, disulfide isomerases could potentially also trigger the coagulation start during pathologic fibrin formation. They could thus represent novel targets for the treatment of thrombotic disorders. The anticoagulant action provided by PDI inhibition is expected to preferentially target the enzyme that is exposed at the lesion area. Since inhibition of PDI decreases fibrin formation also under conditions of diminished platelet adhesion, therapeutic interventions are potentially suitable for an extended range of thrombotic diseases with varying participations of platelets. Nonetheless, additional studies are required to determine the effect of PDI inhibition in further thrombosis models and on hemostasis.

\section{Methods}

Cells and microparticles. Human platelets and human monocytes were isolated as detailed earlier (9). Monocyte TF expression was induced by a 4-hour stimulation with $10 \mathrm{ng} / \mathrm{ml}$ LPS at $37^{\circ} \mathrm{C}$. To generate microparticles in vitro, the isolated monocytes $\left(3 \times 10^{6}\right.$; suspended in RPMI) were activated by LPS (14 hours; $10 \mu \mathrm{g} / \mathrm{ml}$ LPS). The microparticles were prepared from the cell-free supernatants by centrifugation at $2,260 \mathrm{~g}$ ( 20 minutes), followed by a second centrifugation at $17,750 \mathrm{~g}$ (30 minutes). The microparticles were suspended in resuspension buffer $(138 \mathrm{mM} \mathrm{NaCl}, 2.7 \mathrm{mM} \mathrm{KCl}$, $12 \mathrm{mM} \mathrm{NaHCO}_{3}, 0.4 \mathrm{mM} \mathrm{NaH}_{2} \mathrm{PO}_{4}, 1 \mathrm{mM} \mathrm{MgCl}_{2}, 5 \mathrm{mM}$ glucose, $5 \mathrm{mM}$ HEPES; pH 7.35). Only freshly prepared microparticle suspensions were 

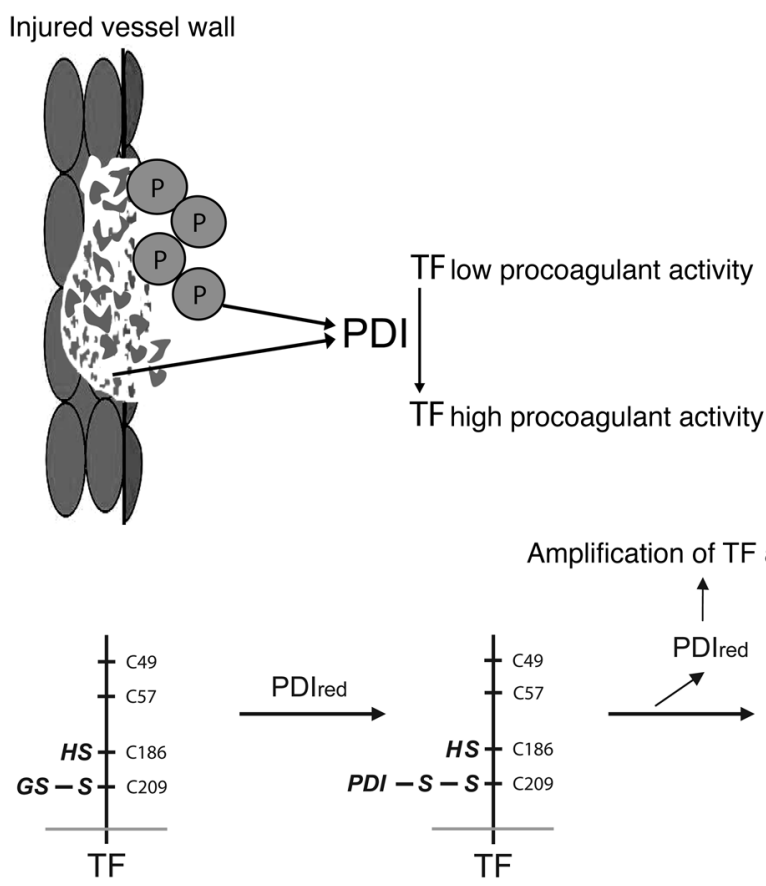

Amplification of TF activation

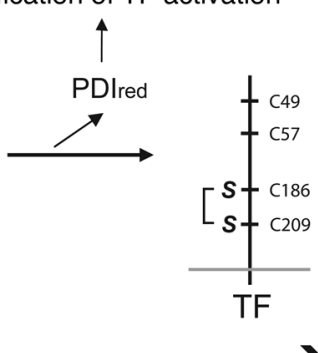

\section{Figure 9}

Model for the activation of TF-dependent coagulation start by PDI. Top: PDI exposure at the vessel injury site contributes to enable the initial step of the extrinsic pathway of blood coagulation, conversion of TF from the functionally inactive to the active form. After vessel injury, PDI can be secreted by activated platelets $(P)$ adhering at the injury site and released from cells of the damaged vessel wall. The exposed PDI might interact with TF expressed by vessel wall cells and TF carrying blood components such as microparticles rapidly recruited to the vessel lesion. In parallel, the exposed PDI can directly contribute to amplify platelet activation. Bottom: Hypothetical molecular mechanism of TF activation by PDI. TF-dependent fibrin formation is low when the Cys186/Cys209 pair of TF is glutathionylated and/or in the free thiol form. Mixed disulfides with glutathione (and other types of linkages) at Cys209 are a constitutive redox form of the protein. PDI can cleave the mixed disulfides of TF when its redox-active cysteines are in the reduced form. This could potentially result in the formation of an unstable PDI-TF intermediate. Through nucleophilic attack by the vicinal free thiol, the intermediate might be disintegrated and be converted into a stable disulfide. The isomerization reaction proposed is thus in principle similar to the PDI-catalyzed isomerization suggested to mediate the correct positioning of protein disulfides in the ER (21). After completing the redox exchange, the same PDI molecule can potentially stimulate additional TF molecules, which would amplify TF activation.

Low procoagulant activity

High procoagulant activity

used, and the time period until their injection into mice (in $250-\mu 1$ portions) was less than 2 hours. Microparticle numbers were assessed by measuring the volume of microparticle suspensions corresponding to 10,000 events with a diameter between 0.1 and $0.8 \mu \mathrm{m}$ (9).

TF and PDI variants. sTF 1-214 was expressed in E. coli and purified under the same conditions as previously described (41). sTF was analyzed by circular dichroism using CDNN software for spectra deconvolution (42). The circular dichroism spectrum of STF thus obtained closely resembled the spectra previously obtained for sTF (43). The contents of secondary structure elements after deconvolution of the spectrum (spectral region 200-260 nm: 7.6\% [ $\alpha$-helical], 28.7\% [antiparallel $\beta$-sheet], 4.5\% [parallel $\beta$-sheet], $28.2 \%$ [ $\beta$-turn], and $39.0 \%$ [random coil]) were similar to those deduced from the $\mathrm{x}$-ray structure of TF and to previously published work (43). DTT (1 mM) did not modify the secondary structure of sTF. sTF $(0.7 \mu \mathrm{g} / \mathrm{ml})$ supported low TF activity $(0.01 \mu \mathrm{M}$ of factor Xa generation; 2-stage assay). sTF was found to contain $0.98 \mathrm{~mol}$ of free thiols $/ \mathrm{mol} \mathrm{sTF}$ as measured by DTNB at $412 \mathrm{~nm}$ in Na-phosphate $(80 \mathrm{mM}, \mathrm{pH} 7.1$; extinction coefficient $38,390 \mathrm{M}^{-1} \mathrm{~cm}^{-1}$; Ellman's assay). Given that the N-terminal cysteines are present as intramolecular disulfide (see below), this indicated that $49 \%$ of the membrane-proximal cysteine pair of sTF was in the reduced form. MPB (0.1 mM; Molecular Probes) decreased the free thiols of sTF by $80 \%$. Other work indicates that sTF $1-218$ is largely devoid of free thiols $(26,33)$. We assume that the differences between those results and our findings are due to the shorter length of sTF used in the present study and/or due to subtle differences in the conditions used to prepare sTF. Mature human PDI was expressed in E. coli and subsequently isolated (44). Seventy to eighty percent of the redox active cysteines of native PDI were in the reduced form (extinction coefficient $14,150 \mathrm{M}^{-1} \mathrm{~cm}^{-1}$ ). In parallel with native PDI, the mutant with Cys to Ser substitutions in the CGHC motifs of the a and a' domains was isolated (PDI $\Delta$ C1,2) (44).

TF-associated free thiols. STF and cells were incubated under varying conditions before labeling of free thiols with MPB. To assess the effect of plate- let releasates on TF, platelets were activated with collagen $(8 \mu \mathrm{g} / \mathrm{ml})$ for 30 minutes and the supernatant was recovered and freed from microparticles by centrifugation. The releasate was incubated for 15 minutes with sTF. Then the samples were treated for 30 minutes with MPB $(0.1 \mathrm{mM})$, and the reaction was terminated by the addition of GSH $(0.2 \mathrm{mM} ; 30 \mathrm{~min}$ utes). Labeled cells were washed 3 times with PBS containing protease inhibitor and phosphatase inhibitor cocktails (Roche). Then the samples were sonicated in ice-cold lysis buffer (50 mM Tris, $0.15 \mathrm{M} \mathrm{NaCl}$; pH 8.0; also containing $1 \%$ Triton X-100 and 5 mM EDTA). Streptavidin-agarose beads (Sigma-Aldrich) were incubated with sTF or cell lysates overnight on a rotating wheel to isolate the biotin-labeled proteins. The streptavidin-agarose beads were washed 3 times with lysis buffer, and the biotinlabeled proteins were released from the beads by boiling in SDS sample buffer (Laemmli buffer) for 3 minutes under nonreducing conditions. Samples were resolved on SDS-PAGE (7.5\% and 16.5\%) under nonreducing conditions and transferred to nitrocellulose membrane (Amersham Biosciences). After electroblotting, the membranes were exposed to the monoclonal anti-hTF antibody (VIC12; ref. 45) and a horseradish peroxidase-conjugated anti-mouse IgG. The experiments shown are representative of a total of 3-4 experiments.

Immunoprecipitation. For the detection of the constitutive glutathionylation of TF, solubilized monocytes were centrifuged 3 times at 3,000 $\mathrm{g}$ for 2 minutes at $4^{\circ} \mathrm{C}$, and the supernatant was incubated with the monoclonal anti-GSH antibody (Virogen; or control IgG) overnight at $4^{\circ} \mathrm{C}$. In other experiments, intact monocytes were incubated with anti-GSH antibody for 60 minutes at room temperature. Then, protein A Sepharose (SigmaAldrich; $50 \mu \mathrm{l}$ ) was added and the mixture incubated for 60 minutes at $4{ }^{\circ} \mathrm{C}$. Immunoprecipitated complexes were collected by centrifugation at $3,000 \mathrm{~g}$ for 2 minutes at $4^{\circ} \mathrm{C}$. After washing 3 times with PBS, the pellets were incubated in SDS sample buffer (without reducing reagents) for 60 minutes at $20^{\circ} \mathrm{C}$. After centrifugation, the supernatant was subjected to SDS-PAGE (7.5\%), followed by electroblotting onto the nitrocellulose membrane. The 
membranes were then exposed to a monoclonal anti-TF antibody (or control IgG), followed by a horseradish peroxidase-conjugated anti-mouse IgG. The experiments were repeated 3 times.

TF glutathionylation. For the labeling of GSH with biotin, equimolar amounts $(10 \mathrm{mM})$ of NHS-biotin (Pierce Biotechnology) and GSH dissolved in PBS were incubated at room temperature. The reaction was terminated after 1 hour, the residual biotin being quenched with ethanolamine $(50 \mathrm{mM})$. To enable the protein incorporation of the labeled glutathione, the cells (or sTF) were incubated for 30-60 minutes with biotin-GSH (5 mM). Subsequently, the cells were washed and solubilized with $1 \%$ Triton X-100 in PBS. sTF was dialyzed using centricon filters. Then streptavidin beads (Sigma) were added to the biotinylated proteins of the cells (or STF) and the mixture was incubated overnight. After sedimentation of the beads, they were washed twice with PBS containing $0.2 \%$ Triton X-100. The proteins were eluted in SDS sample buffer, separated by SDS-PAGE, transferred to nitrocellulose membranes, and the monoclonal anti-TF antibody was employed to detect the glutathionylated protein.

Mass spectrometry. A $20-\mu \mathrm{l}$ aliquot of sTF $(0.13 \mathrm{mg} / \mathrm{ml})$ was digested with $1 \mu \mathrm{g}$ trypsin overnight. For double digests, the protein digestion mixture was spiked with another $1 \mu \mathrm{g}$ trypsin for another 24 hours. Generated peptides were subjected to LC-MS/MS analysis using LTQFT (Thermo Finnigan) or QTOF (Waters) mass spectrometry. The LTQFT hybrid mass spectrometer system consisted of a Waters CapLC and an LTQFT. Data were acquired in data-dependent mode with XCalibur 2.0 and processed with BioWorks. After digestion, the peptides were concentrated on a Waters Symmetry C18 trap column and, after a 5 minute delay, were directed onto a Vydac C18 column VC-10-18W-150 (10 cm × $150 \mu \mathrm{m}$ i.d., $5 \mu \mathrm{m}$, $300 \AA ̊$ A pore size; MicroTech Scientific) for separation. Solvent A was $0.2 \%$ formic acid in water and solvent $B$ was $0.2 \%$ formic acid in acetonitrile. The separation was started for the first 5 minutes with $95 \%$ solvent $A$, then a gradient was ramped from $5 \%$ to $35 \%$ B within 10 minutes. Solvent B was then ramped up to $90 \%$ over 10 minutes and held at $90 \%$ for $10 \mathrm{~min}$ utes. Then the starting conditions were restored to equilibrate the column. The flow was $6 \mu \mathrm{l} / \mathrm{min}$ in a split ratio of $1: 10$. Columns were kept at room temperature. The QTOF2 hybrid mass spectrometer system consisted of a nanoAcquity UPLC and a QTOF2. Data were acquired in a data-dependent mode and processed with MassLynx 4.0 using a similar setup as described above. The peptide fragment data obtained from the LTQFT were processed using BioWorks to create data files that were merged using a PERL program (provided by MatrixScience and modified in our laboratory) for subsequent database searches. QTOF data were processed accordingly using MassLynx. A Mascot search engine was used for database searches, applying the following parameters: SwissProt database, charge states +2 and $+3,1$ missed cleavage. A window of 5 ppm mass accuracy for precursor ions and 0.6 Da accuracy for MS/MS data was chosen for the LTQFT data. For QTOF data, a window of 0.3 Da mass accuracy for precursor ions and for MS/MS data was chosen. Probability-based MASCOT scores were considered significant when greater than the cutoff score, indicating either identity or homology $(P<0.05)$ for individual ions.

Procoagulant activity. TF-expressing cells, platelets and microparticles were incubated for different time intervals. To neutralize the TF activity of monocyte microparticles, a monoclonal anti-hTF antibody (VIC7; ref. 46) was used. To assess whether microparticle TF was responsible for the TF activity in suspensions of activated platelets and microparticles, isolated microparticles and activated platelets (10-minute stimulation with collagen type I, $8 \mu \mathrm{g} / \mathrm{ml}$ ) were separately incubated with anti-TF antibody (VIC7). Then, microparticles and platelets were centrifuged (at 17,750 g and $330 \mathrm{~g}$, respectively), the supernatants were removed to eliminate free antibody, and the blood components were suspended in resuspension buffer. In separate samples, bacitracin (6 mM; Sigma-Aldrich), and anti-PDI (mab3-019; or isotype control antibody, both diluted 1:70; Affinity Bioreagents) were added. To prepare cell lysates, human monocytes (for the experiments shown in Figure 6C) or CHO cells were suspended in $30 \mu \mathrm{l}$ of PBS containing $15 \mathrm{mM}$ of octyl- $\beta$-D-glucopyranoside (Sigma-Aldrich), frozen at $-80^{\circ} \mathrm{C}$ for 15 minutes, and thawed. TF activity was determined using a 2 -stage assay in the presence of FVIIa ( $2 \mathrm{nM}$; Novo Nordisk) and $\mathrm{CaCl}_{2}(25 \mathrm{mM})$ for 15 minutes at room temperature, followed by addition of FX (200 nM; Calbiochem) for a further 30 minutes at room temperature. When $\mathrm{CaCl}_{2}$ concentrations were reduced to $8 \mathrm{mM}$, TF activity was stimulated by PDI in a way comparable to that used at higher $\mathrm{CaCl}_{2}$ concentrations. TF activity was also measured using a coagulation factor concentrate as previously described (9). S2222 was employed to assess the amount of factor Xa generated. The method employs a synthetic substrate specific for factor $\mathrm{Xa}$, the product of hydrolysis being directly proportional to the amount of factor Xa present.

Animals. WT mice (SV129S1) were obtained from Charles River. Low-hTF mice and mice harboring hTF (both on a C57BL/6 background) were generated as previously described $(23,24)$. The mice used were age matched and did not differ in carotid arterial diameter and thickness of the vessel wall.

In vivo model of intravascular coagulation by carotid artery ligation. For injury of the carotid artery (18), mice were anesthetized by intraperitoneal injection of a solution of midazolame ( $5 \mathrm{mg} / \mathrm{kg}$ body weight; Ratiopharm), medetomidine $(0.5 \mathrm{mg} / \mathrm{kg}$ body weight; Pfizer), and fentanyl $(0.05 \mathrm{mg} / \mathrm{kg}$ body weight; CuraMed Pharma). The common carotid artery was dissected free, and endothelial denudation was induced near the carotid bifurcation by ligation of the vessel until complete cessation of the blood flow occurred. The ligation was removed after 5 minutes. All procedures performed on mice were approved by the local legislation on protection of animals (Regierung von Oberbayern, Munich, Germany). Fibrin formation was visualized by high-speed intravital microscopy by a modification of a previous published technique (47). To detect fibrin in vivo, a monoclonal antibody against the fibrin II $\beta$ chain (B $\beta$ 15-42, Clone T2G1; Accurate Chemical) was labeled with Alexa Fluor 488 using the Alexa Fluor Protein Labeling Kit (Molecular Probes) according to the manufacturer's instructions and infused intravenously ( $2 \mathrm{mg} / \mathrm{kg}$ body weight). Isotype-matched irrelevant IgG (mouse IgG2a; Sigma-Aldrich) was used as a negative control. Fibrin formation is presented as absolute fluorescence intensity (arbitrary units). To evaluate the contribution of the microparticles for fibrin formation in vivo, monocyte microparticles $\left(10^{7}\right.$, suspended in $\left.250 \mu \mathrm{l}\right)$ were infused into the jugular vein. The residual amounts of LPS present in the preparations of washed microparticles alone do not account for the rapid increase in fibrin formation after microparticle infusion. In some experiments, mice were treated with anti-PDI and control antibody (800 $\mu \mathrm{g} /$ animal; mab3-019 or respective control antibody; Affinity Bioreagents) or with PDI (200 $\mu \mathrm{g} / \mathrm{animal})$. Endothelial disruption was induced, triggering local platelet adhesion and aggregation. In parallel experiments, the microparticles were labeled with 5-carboxyfluorescein diacetate succinimidyl ester (DCF) before inclusion into the blood circulation of mice pretreated with the anti-PDI antibody (or control $\operatorname{IgG}$ ). Local microparticle accumulation was determined by intravital microscopy. Where indicated, platelets were differentially tagged with rhodamine $6 \mathrm{G}$ and infused together with the labeled microparticles to directly assess the interaction between the 2 blood components. In separate experiments, the requirement of platelets for the PDI-evoked fibrin formation was evaluated. Mice were pretreated with $6 \mathrm{mg} / \mathrm{kg}$ of monoclonal rat anti-mouse GPVI antibody (IgG2a, clone IF5-1-11; kindly provided by Elisabeth Kremmer, GSF Research Center of Environment and Health, Munich, Germany). The specificity of the antibody was verified by its ability to recognize a single band at the expected molecular weight of GPVI in murine platelets. Alternatively, a mixture of anti-GPIb $\alpha$ antibodies $(50 \mu \mathrm{g} / \mathrm{mouse}, \mathrm{R} 300$; 
Emfret Analytics) was infused into mice to decrease platelet adhesion via induction of thrombocytopenia (22). Fibrin formation was subsequently determined in the presence of the anti-PDI antibody (or control IgG) by intravital microscopy. For detection of PDI in vivo by intravital microscopy, an Alexa Fluor 488-labeled anti-PDI antibody (H-160; Santa Cruz Biotechnology Inc.) or control IgG was injected into the murine blood circulation $(20 \mu \mathrm{g} /$ mouse $)$.

Activation of in vivo coagulation by wire-induced vascular injury. Wire-induced endothelial disruption was performed as previously described (18).

Tail bleeding. Tails of WT mice were cut $5 \mathrm{~mm}$ from the tip with a scalpel, and the time was measured until bleeding stopped using a paper filter.

Detection of fibrin ex vivo. Thirty minutes after induction of carotid injury, the arteries were excised, gently flushed with PBS to remove residual blood, and immediately frozen using liquid nitrogen. Segments $(2 \mathrm{~mm})$ of injured vessels or control vessels were homogenized and lysed in a buffer containing protease inhibitors. Proteins were separated on SDS-PAGE (4\%-20\%) and transferred to nitrocellulose membranes. This was followed by incubation with mouse anti-fibrin II $\beta$ chain antibody (B $\beta$ 15-42, clone T2G1; Accurate Chemical; $2 \mu \mathrm{g} / \mathrm{ml}$ ) and treatment with horse radish peroxidasetagged anti-mouse IgG $\mathrm{H}+\mathrm{L}$ (Vector, $0.5 \mu \mathrm{g} / \mathrm{ml}$ ). Finally, the membranes were developed by chemiluminescence (PerkinElmer).

Intravital imaging. We used a high-speed widefield Olympus BX51WI fluorescence microscope with a long-distance condenser and a $\times 20$ (NA 0.95 ) water-immersion objective for intravital imaging of fibrin formation, platelet adhesion and microparticle recruitment. The digital microscope allows near-simultaneous collection of images in 3 separate channels, each monitoring a different fluorescence emission. The system is equipped with an Olympus MT 20 monochromator for excitation and a F-View CCD camera (Olympus). A Dell workstation with the Olympus Cell/R software was used for synchronization of components, data acquisition, and image analysis.

Immunohistochemistry. Fifteen minutes following ligation of the murine carotid artery, injured vessel sections were excised. Sections of the vessels were exposed to Alexa Fluor 594-labeled rat anti-CD41 antibody (BD Bio- sciences) and to Alexa Fluor 488-labeled anti-PDI antibody (H-160; Santa Cruz Biotechnology Inc.).

Statistics. All data shown represent means $\pm \mathrm{SD}$, unless otherwise indicated. Measurements performed under identical conditions and time points were analyzed by Mann-Whitney rank sum test. Consecutive measurements were compared by ANOVA repeated measurements. $P<0.05$ was considered statistically significant.

\section{Acknowledgments}

We thank Hans-Dieter Söling, Manuel Than, Rein Aasland, and Klaus T. Preissner for discussions and helpful suggestions. We thank Elisabeth Kremmer, Viktor Magdolen, Sybille Albrecht, and Thomas Luther for providing substances. C. Reinhardt, D. Manukyan, and B. Altmann have been participants of the DFGGraduiertenkolleg "Vaskuläre Biologie in der Medizin”. L. Grahl was supported by DFG-Forschergruppe "Prävention des Ischämie-Reperfusionsschadens". S. Massberg is a Heisenberg fellow of the DFG. This work was supported by grants from the DFG and Stiftung für Pathobiochemie und Molekulare Diagnostik (to B. Engelmann and S. Massberg), the Intramural Research Program of the NIH NIDDK, and the Betty and Gordon Moore Foundation (to S. Hess). Mass spectrometry experiments were conducted at the Proteomics and Mass Spectrometry Facility of the NIDDK.

Received for publication April 11, 2007, and accepted in revised form December 19, 2007.

Address correspondence to: Bernd Engelmann, Institut für Klinische Chemie, Ludwig-Maximilians-Universität, Marchioninistr. 15, 81377 Munich, Germany. Phone: 49-89-7095-3243; Fax: 4989-7095-6220; E-mail: Bernd.Engelmann@med.uni-muenchen.de.

Christoph Reinhardt and Marie-Luise von Brühl contributed equally to this work.
1. Dahlback, B. 2000. Blood coagulation. Lancet. 355:1627-1632.

2. Schenone, M., Furie, B.C., and Furie, B. 2004. The blood coagulation cascade. Curr. Opin. Hematol. 11:272-277.

3. Gomez, K., and McVey, K.H. 2006. Tissue factor initiated blood coagulation. Front. Biosci. 11:1349-1359.

4. Mackman, N., Tilley, R.E., and Key, N.S. 2007. Role of the extrinsic pathway of blood coagulation in hemostasis and thrombosis. Arterioscler. Thromb. Vasc. Biol. 27:1687-1693.

5. Steffel, J., Lüscher, T.F., and Tanner, F.C. 2006. Tissue factor in cardiovascular diseases: molecular mechanisms and clinical implications. Circulation. 113:722-731.

6. Maynard, J.R., Heckman, C.A., Pitlick, F.A., and Nemerson, Y. 1975. Association of tissue factor activity with the surface of cultured cells. J. Clin. Invest. 55:814-824.

7. Bach, R., and Moldow, C.F. 1997. Mechanism of tissue factor activation on HL-60 cells. Blood. 89:3270-3276

8. Bach, R.R. 2006. Tissue factor encryption. Arterioscler. Thromb. Vasc. Biol. 26:456-461.

9. Muller, I., et al. 2003. Intravascular tissue factor initiates coagulation via circulating microvesicles and platelets. FASEB J. 17:476-478.

10. Mandal, S.K., Iakhiaev, A., Pendurthi, U.R., and Rao, L.V.M. 2005. Acute cholesterol depletion impairs functional expression of tissue factor in fibroblasts: modulation of tissue factor activity by membrane cholesterol. Blood. 105:153-160.

11. Wolberg, A.S., Monroe, D.M., Roberts, H.R., and
Hoffman, M.R. 1999. Tissue factor de-encryption: ionophore treatment induces changes in tissue factor activity by phosphatidylserine-dependent and -independent mechanisms. Blood Coagul. Fibrinolysis. 10:201-210.

12. Ahamed, J., et al. 2006. Disulfide isomerization switches tissue factor from coagulation to cell signaling. Proc. Natl. Acad. Sci. U. S. A. 103:13932-13937.

13. Mallat, Z., et al. 1999. Shed membrane microparticles with procoagulant potential in human atherosclerotic plaques: a role for apoptosis in plaque thrombogenicity. Circulation. 99:348-353.

14. Falati, S., et al. 2003. Accumulation of tissue factor into developing thrombi in vivo is dependent upon microparticle P-selectin glycoprotein ligand 1 and platelet P-selectin. J. Exp. Med. 197:1585-1598.

15. Hrachovinová, I., et al. 2003. Interaction of P-selectin and PSGL-1 generates microparticles that correct hemostasis in a mouse model of hemophilia A. Nat. Med. 9:1020-1025.

16. Engelmann, B. 2006. Initiation of coagulation by tissue factor carriers in blood. Blood Cells Mol. Dis. 36:188-190.

17. Essex, D.W., Li, M., Miller, A., and Feinman, R.D 2001. Protein disulfide isomerase and sulfhydryldependent pathways in platelet activation. Biochemistry. 40:6070-6075.

18. Massberg, S., et al. 2003. A crucial role of glycoprotein VI for platelet recruitment to the injured arterial wall in vivo. J. Exp. Med. 197:41-49.

19. Biró, E., et al. 2003. Human cell-derived microparticles promote thrombus formation in vivo in a tissue factor-dependent manner. J. Thromb. Haemost.
1:2561-2568.

20. Tu, B.P., and Weissman, J.S. 2004. Oxidative protein folding in eukaryotes: mechanisms and consequences. J. Cell Biol. 164:341-346.

21. Wilkinson, B., and Gilbert, H.F. 2004. Protein disulfide isomerase. Biochim. Biophys. Acta. 1699:35-44.

22. Iannacone, M., et al. 2005. Platelets mediate cytotoxic T lymphocyte-induced liver damage. Nat. Med. 11:1167-1169.

23. Pawlinski, R., et al. 2007. Role of cardiac myocyte tissue factor in heart hemostasis. J. Thromb. Haemost. 5:1693-1700.

24. Parry, G.C.N., Erlich, J.H., Carmeliet, P., Luther, T., and Mackman, N. 1998. Low levels of tissue factor are compatible with development and hemostasis in mice. J. Clin. Invest. 101:560-569.

25. Ingalls, H.M., Goodloe-Holland, C.M., and Luna, E.J. 1986. Junctional plasma membrane domains isolated from aggregating Dictyostelium discoideum amebae. Proc. Natl. Acad. Sci. U. S. A. 83:4779-4783.

26. Harlos, K., et al. 1994. Crystal structure of the extracellular region of human tissue factor. Nature. 370:662-666.

27. Banner, D.W., et al. 1996. The crystal structure of the complex of blood coagulation factor VIIa with soluble tissue factor. Nature. 380:41-46.

28. Ghezzi, P. 2005. Regulation of protein function by glutathionylation. Free Radic. Res. 39:573-580.

29. Jessop, C.E., Chakravarthi, S., Watkins, R.H., and Bulleid, N.J. 2004. Oxidative protein folding in the mammalian endoplasmic reticulum. Biochem. Soc. Trans. 32:655-658.

30. Peltoniemi, M.J., Karala, A.-R., Jurvansuu, J.K., Kin- 
nula, V.L., and Ruddock, L.W. 2006. Insights into deglutathionylation reactions. Different intermediates in the glutaredoxin and protein disulfide isomerase catalyzed reactions are defined by the gamma-linkage present in glutathione. J. Biol. Chem. 281:33107-33114.

31. Turano, C., Coppari, S., Altieri, F., and Ferraro, A. 2002. Proteins of the PDI family: unpredicted non-ER locations and functions. J. Cell Physiol. 193:154-163.

32. Park, B., et al. 2006. Redox regulation facilitates optimal peptide selection by MHC class I during antigen processing. Cell. 127:369-382.

33. Versteeg, H.H., and Ruf, W. 2007. Tissue factor coagulant function is enhanced by protein disulfide isomerase independent of oxido-reductase activity. J. Biol. Chem. 282:25416-25424.

34. Ellgaard, L., and Ruddock, L.W. 2005. The human protein disulphide isomerase family: substrate interactions and functional properties. EMBO Rep. 6:28-32.

35. Hogg, P.J. 2003. Disulfide bonds as switches for protein function. Trends Biochem. Sci. 28:210-214.
36. Matthias, L.J., et al. 2002. Disulfide exchange in domain 2 of CD4 is required for entry of HIV-1. Nat. Immunol. 3:727-732.

37. Patel, S.D., Rajala, M.W., Rossetti, L., Scherer, P.E., and Shapiro, L. 2004. Disulfide-dependent multimeric assembly of resistin family hormones. Science. 304:1154-1158.

38. Wang, W., et al. 2005. Reversible silencing of CFTR chloride channels by glutathionylation. J. Gen. Physiol. 125:127-141.

39. Ruggeri, Z.M. 2002. Platelets in atherothrombosis. Nat. Med. 8:1227-1234.

40. Libby, P., and Theroux, P. 2005. Pathophysiology of coronary artery disease. Circulation. 111:3481-3488.

41. Albrecht, S., et al. 2002. Soluble tissue factor interferes with angiostatin-mediated inhibition of endothelial cell proliferation by lysine-specific interaction with plasminogen kringle domains. Thromb. Haemost. 88:1054-1059.

42. Böhm, G., Muhr, R., and Jaenicke, R. 1992. Quantitative analysis of protein far UV circular dichroism spectra by neuronal networks. Protein Eng. 5:191-195.
43. Andersson, D., Carlsson, U., and Freskgård, P.-O. 2001. Contribution of tryptophan residues to the CD spectrum of the extracellular domain of human tissue factor: application in folding studies and prediction of secondary structure. Eur. J. Biochem. 268:1118-1128.

44. Winter, J., Klappa, P., Freedman, R.B., Lilie, H., and Rudolph, R. 2002. Catalytic activity and chaperone function of human protein-disulfide isomerase are required for the efficient refolding of proinsulin. J. Biol. Chem. 277:310-317.

45. Muller, M., et al. 1999. Localization of tissue factor in actin-filament-rich membrane areas of epithelial cells. Exp. Cell Res. 248:136-147.

46. Leon, C., et al. 2004. Platelet ADP receptors contribute to the initiation of intravascular coagulation. Blood. 103:594-600.

47. Falati, S., Gross, P., Merrill-Skoloff, G., Furie, B.C., and Furie, B. 2002. Real-time in vivo imaging of platelets, tissue factor and fibrin during arterial thrombus formation in the mouse. Nat. Med. 8:1175-1181. 